\title{
Expression of orotate phosphoribosyltransferase in colorectal carcinoma: An immunohistochemical analysis in several components of neoplastic lesions
}

\author{
YUICHI SANADA ${ }^{1,2}$, KAZUHIRO YOSHIDA $^{1}$, JUN HIHARA $^{1}$ and MORIHITO OKADA ${ }^{1}$ \\ ${ }^{1}$ Department of Surgical Oncology, Research Institution for Radiation \\ Biology and Medicine, Hiroshima University, Hiroshima, Japan
}

Received April 9, 2008; Accepted May 15, 2008

DOI: 10.3892/or_00000102

\begin{abstract}
The purpose of this study was to evaluate the pattern of the expression of orotate phosphoribosyltransferase (OPRT) in several components of colorectal carcinoma (CRC). Fifty-six surgically-resected samples of CRC were subjected to immunohistochemistry with a polyclonal anti-OPRT antibody. Grading was performed independently for several components of CRC, including mucosal carcinoma lesions $(n=56)$, infiltrative lesions $(\mathrm{n}=53)$, lymphovascularly invasive lesions $(n=34)$ and metastatic lymph nodes $(n=17)$. The expression of OPRT in mucosal carcinoma and infiltrative lesions correlated significantly only with the presence of lymphovascular invasion ( $\mathrm{p}=0.0007$ and $<0.0001$, respectively). The frequency of OPRT expression in mucosal carcinoma, infiltrative and lymphovascularly invasive lesions as well as metastatic lymph nodes was $32.1,69.8,88.2$ and $88.8 \%$, respectively. In addition, nuclear staining of OPRT was observed in metastatic lymph nodes and lymphovascularly invasive lesions. Our results suggest that OPRT is involved in the invasion and metastasis of CRC.
\end{abstract}

\section{Introduction}

Orotate phosphoribosyltransferase (OPRT) is an essential enzyme for the activation of 5-fluorouracil (5-FU) (1). Several studies describing the expression or activity of OPRT in gastrointestinal carcinoma have been published $(2,3)$. Since OPRT is involved in the first step of 5-FU activation, previous studies have focused on the association between OPRT and chemosensitivity $(4,5)$. Most researchers concluded that colorectal carcinoma (CRC) patients expressing high levels

Correspondence to: Dr Yuichi Sanada, ${ }^{2}$ Present address: Department of Surgical Oncology, Gifu University School of Medicine, 1-1 Yanagido, Gifu City 501-1194, Gifu, Japan

E-mail: ysanadasurg@hotmail.com

Key words: orotate phosphoribosyltransferase, claudin-1, colorectal carcinoma of OPRT have higher 5-FU sensitivity and a better prognosis than those expressing lower levels of OPRT $(4,5)$. Tokunaga et al (4) reported that OPRT expression is associated with a high sensitivity for postoperative adjuvant chemotherapy. However, the association between OPRT and colorectal carcinogenesis, invasion and metastasis is unclear. To gain a better understanding of the function of OPRT in CRCs, further morphological description is needed. Therefore, in the present study, we used immunohistochemistry (IHC) to analyze 56 resected CRCs with an individual grading for several tumor components, including mucosal carcinoma, infiltrative and lymphovascularly invasive lesions as well as metastatic lymph nodes.

\section{Materials and methods}

Cases. The study group comprised of 56 patients (38 men and 18 women; mean age, 64.7 years; range: $32-92$ years) whose CRCs were classified as Stage I to IV according to the World Health Organization classification and were resected surgically between 1999 and 2003 at the Department of Surgical Oncology, Hiroshima University. Clinicopathological characteristics of the 56 patients are summarized in Table I. No patient received chemotherapy or radiation prior to resection. Stage IV patients $(n=4)$ had peritoneal invasion near the tumor that was resectable.

Histopathological evaluation. Representative paraffinembedded tissue blocks from each case were obtained. Hematoxylin and eosin (H\&E)-stained sections from each case were screened by light microscopy. A histopathological examination of the 56 CRCs included an assessment of the depth of invasion and presence of lymphovascular invasion as well as lymph node metastasis. In the present study, a histological subtype was determined separately in mucosal and infiltrative components deeper than the muscular propria. Mucinous carcinoma was diagnosed when the tumor was composed predominantly of mucinous components defined as a nodular extracellular mucin lake with scanty malignant epithelial cells.

IHC. IHC for OPRT was carried out with a polyclonal antiOPRT antibody (1:1000, Taiho, Tokyo, Japan) and a Dako 
LSAB kit (Dako, Carpinteria, CA, USA). Paraffin-embedded sections were deparaffinized in xylene and rehydrated through a graded ethanol series. After the endogenous peroxidase activity was blocked with $3 \% \mathrm{H}_{2} \mathrm{O}_{2}$ in methanol for $10 \mathrm{~min}$, sections were incubated with a primary antibody for $8 \mathrm{~h}$ at $4{ }^{\circ} \mathrm{C}$ followed by sequential 10 -min incubations with biotinylated anti-rabbit IgG and peroxidase-labeled streptavidin. Staining was completed with a 10-min incubation in a substratechromogen solution. Sections were counterstained in $0.1 \%$ hematoxylin. Slides were examined under a light microscope and scored independently by two examinors (Y.S. and K.Y.). OPRT expression was quantified with the following grading system, which is based on the intensity and frequency of staining and is classified into four grades from 0 to $3^{+}$; where 0 is undetectable staining or weak, patchy cytoplasmic staining; $1^{+}$is focal cytoplasmic staining in $<25 \%$ of tumor cells; $2^{+}$is diffuse, granular cytoplasmic staining in $>50 \%$ of tumor cells and $3^{+}$is diffuse cytoplasmic staining in most tumor cells with dense nuclear staining in certain tumor cells. Grades 0 and $1^{+}$were grouped together and considered as a negative expression and grades $2^{+}$and $3^{+}$were considered as a positive expression. In addition, in the primary tumors, grading was independently performed between mucosal carcinoma lesions $(n=56)$, corresponding infiltrative lesions deeper than muscular propria $(n=53)$ and corresponding lymphovascularly invasive lesions $(n=34)$. For the 25 cases with lymph node metastasis, tissue blocks of the metastatic lymph nodes were available for 17 cases. We also performed OPRT IHC in metastatic lymph nodes and compared the expression patterns in those of corresponding primary lesions.

Statistical analyses. OPRT staining in mucosal carcinoma and infiltrative lesions were characterized independently and compared statistically with clinicopathological factors including $\mathrm{T}$-grade, $\mathrm{N}$-grade and the presence of lymphovascular invasion. Additionally, the frequency (number of cases) of OPRT expression was compared between tumor components (mucosal carcinoma lesions vs. infiltrative lesions, mucosal carcinoma lesions versus lymphovascularly invasive lesions and mucosal carcinoma lesions vs. metastatic lymph nodes). Analyses were performed with SPSS software (Version 10.5, SPSS, Inc., Chicago, IL, USA). A P-value of $<0.05$ was regarded as statistically significant.

\section{Results}

Non-cancerous components do not express OPRT. In corresponding non-neoplastic mucosa, most cells did not express OPRT (Fig. 1A). Granular cytoplasmic staining was observed only in the crypt components (Fig. 1A, arrowhead). Some adenomas showed weak cytoplasmic staining, which was considered negative (Fig. 1B). The cases in which tumor cells were positive for OPRT showed no immunoreaction in corresponding non-neoplastic mucosa (Fig. 1C).

Expression of OPRT in mucosal carcinoma components (Table II). In mucosal carcinoma components, OPRT staining was positive in 18 of the 56 cases (Fig. 1D-G). No case showed nuclear staining. The 18 cases that were positive for OPRT showed diffuse cytoplasmic staining throughout the tumor
Table I. Characteristics of colorectal carcinoma analyzed for OPRT.

Case

Age

$32-92(64.7)$ y

Gender

Male

Female

Tumor location

Colon

Rectum

Lymph node metastasis

$(-)$

Lymphovascular invasion

(+)

(-)

Cancer stage

I

II

III

IV

Histological differentiation

Mucosal component

Well

Moderate

Poor

Infilrative component

Well

Moderate

Poor

Mucinous

7

(Fig. 1E) except for one case with irregular granular staining in $\sim 50 \%$ of the tumor (Fig. 1D). In contrast, the 36 cases negative for OPRT showed no staining in the tumor (Fig. $1 \mathrm{~F}$ and $\mathrm{G}$ ). In addition, there was no significant correlation between OPRT expression and cancer stage, $\mathrm{T}$-grade or $\mathrm{N}$-grade. However, OPRT expression correlated positively with the presence of lymphovascular invasion $(\mathrm{p}=0.0007)$.

Expression of OPRT in infiltrative components (Table III). To assess the pattern of OPRT expression in components infiltrating deeper than the muscular propria, three cases with the T-grade of T1 were excluded. Tumor cells in infiltrative components showed positive staining for OPRT in 37 (69.8\%) of the 53 cases, an expression rate $\sim 2$.2-fold higher than that in mucosal carcinoma components. These 37 cases included 8 in which corresponding mucosal carcinoma lesions did not show a positive staining for OPRT (Fig. 1H-J). Conversely, in 16 cases negative for OPRT in infiltrative components, no case showed a positive staining for OPRT in 
Table II. Relationship between OPRT expression in mucosal components and clinicopathological features $(n=56)$.

\begin{tabular}{cccc}
\hline Variables & $\begin{array}{c}\text { OPRT (-) } \\
\left(0,1^{+}\right)\end{array}$ & $\begin{array}{c}\text { OPRT }(+) \\
\left(2^{+}, 3^{+}\right)\end{array}$ & P-values \\
\hline
\end{tabular}

\begin{tabular}{lrrc}
$\begin{array}{l}\text { Number of } \\
\text { patients }\end{array}$ & 38 & 18 & \\
$\mathrm{~N} \mathrm{(-)}$ & 19 & 6 & 0.267 \\
$\mathrm{~N}(+)$ & 19 & 12 & \\
Ly or V (-) & 19 & 2 & 0.007 \\
Ly or V (+) & 18 & 16 & 0.153 \\
Stage & & & \\
I & 10 & 0 & \\
II & 9 & 5 & \\
III & 16 & 12 & 0.143 \\
IV & 3 & 1 & $($ Ttage I II vs. III IV) \\
T-grade & & & \\
1 & 3 & 0 & \\
2 & 6 & 1 & \\
3 & 19 & 12 & \\
4 & 10 & 5 & \\
Size & & 8 & \\
$<5 \mathrm{~cm}$ & 20 & 10 & \\
$>5 \mathrm{~cm}$ & 18 & & \\
\hline
\end{tabular}

$\mathrm{N}$, the presence of lymph node metastasis; Ly or $\mathrm{V}$, the presence of lymphovascular invasion and $\mathrm{T}, \mathrm{T}$-grade.

corresponding mucosal carcinoma components. OPRT was diffusely expressed to a greater extent, especially in the tumor cells adjacent to the infiltrative margin (Fig. 1I and $\mathrm{K}$ arrowhead). A subset of tumor cells in infiltrative components showed nuclear staining in three cases (Fig. 1L-N, arrowhead). Additionally, in contrast with previous reports, mucinous carcinoma cells in infiltrative components showed diffuse staining for OPRT in 6 of the 7 cases (Fig. 1O-Q). Similar to our results in mucosal carcinoma lesions, OPRT expression in infiltrative components correlated positively only with the presence of lymphovascular invasion $(\mathrm{p}<0.0001)$.

Expression of OPRT in lymphovascularly invasive lesions (Table IV). Histologically, lymphovascular invasion was observed in $34(60.7 \%)$ of the 56 cases. Most tumor cells showed diffuse cytoplasmic staining in $30(88.2 \%)$ of the 34 cases, including $10(33.3 \%)$ cases with nuclear staining (Fig. 1R-T, arrowhead).

Expression of OPRT in metastatic lymph nodes (Table IV). Lymph node metastasis was observed in 25 (44.6\%) of the 56 cases. Paraffin-embedded sections were available for 17 of these cases. OPRT was expressed in metastatic lymph nodes in $15(88.2 \%)$ of the 17 cases. Staining was dense and mainly localized in the cytoplasm, with no staining of surrounding
Table III. Relationship between OPRT expression in infiltrative components and clinicopathological features $(\mathrm{n}=53)$.

\begin{tabular}{cccc}
\hline Variables & OPRT (-) & OPRT $(+)$ & P-values \\
$\left(0,1^{+}\right)$ & $\left(2^{+}, 3^{+}\right)$ &
\end{tabular}

\begin{tabular}{lrrc}
\hline $\begin{array}{l}\text { Number of } \\
\text { patients }\end{array}$ & 16 & 37 & \\
N (-) & 9 & 6 & 0.3 \\
N (+) & 7 & 12 & \\
Ly or V (-) & 11 & 2 & 0.0001 \\
Ly or V (+) & 5 & 29 & \\
Stage & & & 0.132 \\
I & 1 & 6 & \\
II & 8 & 6 & \\
III & 7 & 21 & 0.661 \\
IV & 0 & 4 & $($ T2 vs. T3 T4) \\
T-grade & & & \\
1 & 0 & 0 & \\
2 & 1 & 6 & \\
3 & 11 & 20 & \\
4 & 4 & 11 & 0.077 \\
Size & & & \\
$<5$ cm & 11 & 15 & \\
$>5$ cm & 5 & 22 & \\
Mucosal (-) & 16 & 8 & \\
Mucosal (+) & 0 & 29 & \\
\hline
\end{tabular}

$\mathrm{N}$, the presence of lymph node metastasis; Ly or $\mathrm{V}$, the presence of lymphovascular invasion; T, T-grade; mucosal (-), number of cases in which corresponding mucosal carcinoma lesions did not express OPRT and mucosal (+), number of cases in which corresponding mucosal carcinoma lesions express OPRT.

lymphocytes (Fig. 1U). Two OPRT-negative cases formed mucinous infiltrations (Fig. 1V). In $7(46.6 \%)$ of the 17 cases, corresponding mucosal carcinoma components did not express OPRT (Fig. 1W and X). Nuclear staining was observed in $5(29.4 \%)$ of the 17 cases (Fig. 1Y and Z). Statistically, OPRT immunoreactivity in infiltrative lesions, lymphovascularly invasive components and metastatic lymph nodes was significantly higher than that in mucosal carcinoma lesions (Table V).

Comparison of expression patterns between OPRT and CLDN1 in four cases (Table VI). Nuclear staining of OPRT was observed in metastatic lymph nodes, whereas only cytoplasmic staining was observed in corresponding mucosal carcinoma lesions. Since OPRT is a metabolic enzyme, expression is expected to be localized in the cytoplasm. The mechanism of the mislocalization observed in the present study is unclear. Recently, Dhawan et al (6) reported that the expression of claudin-1 (CLDN1), a tight junction protein, is 

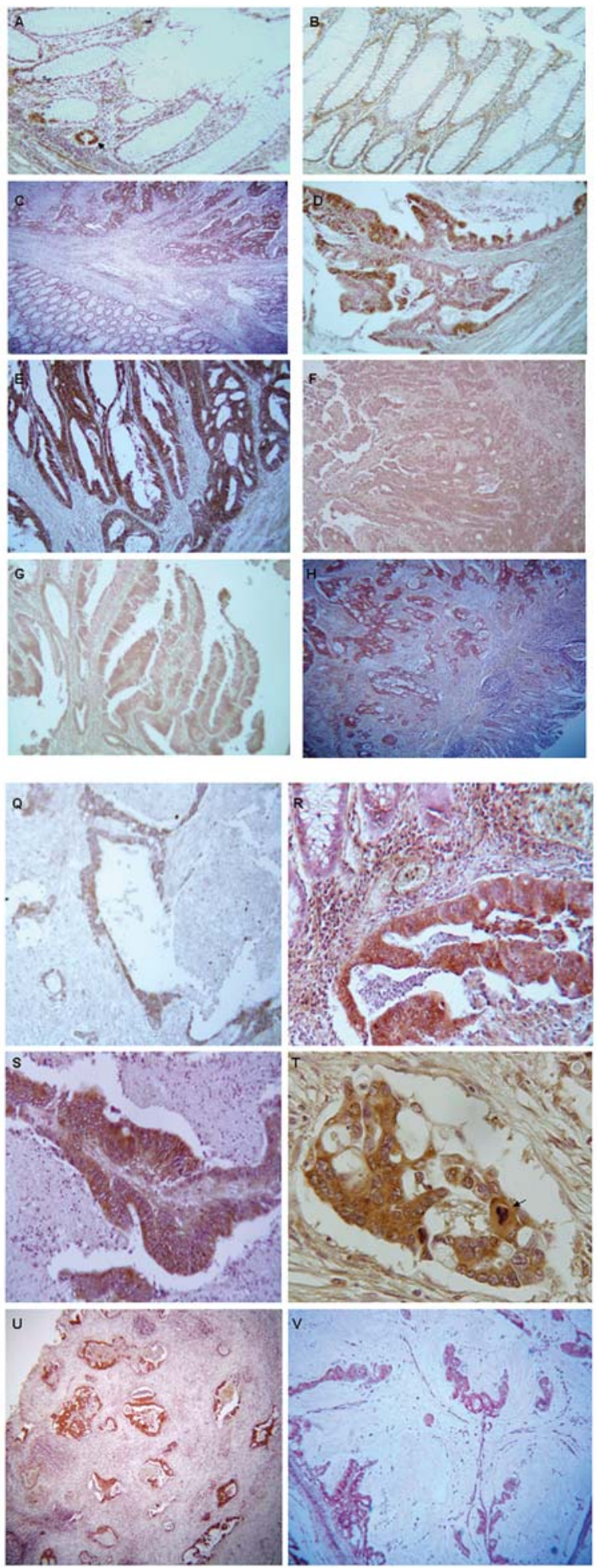
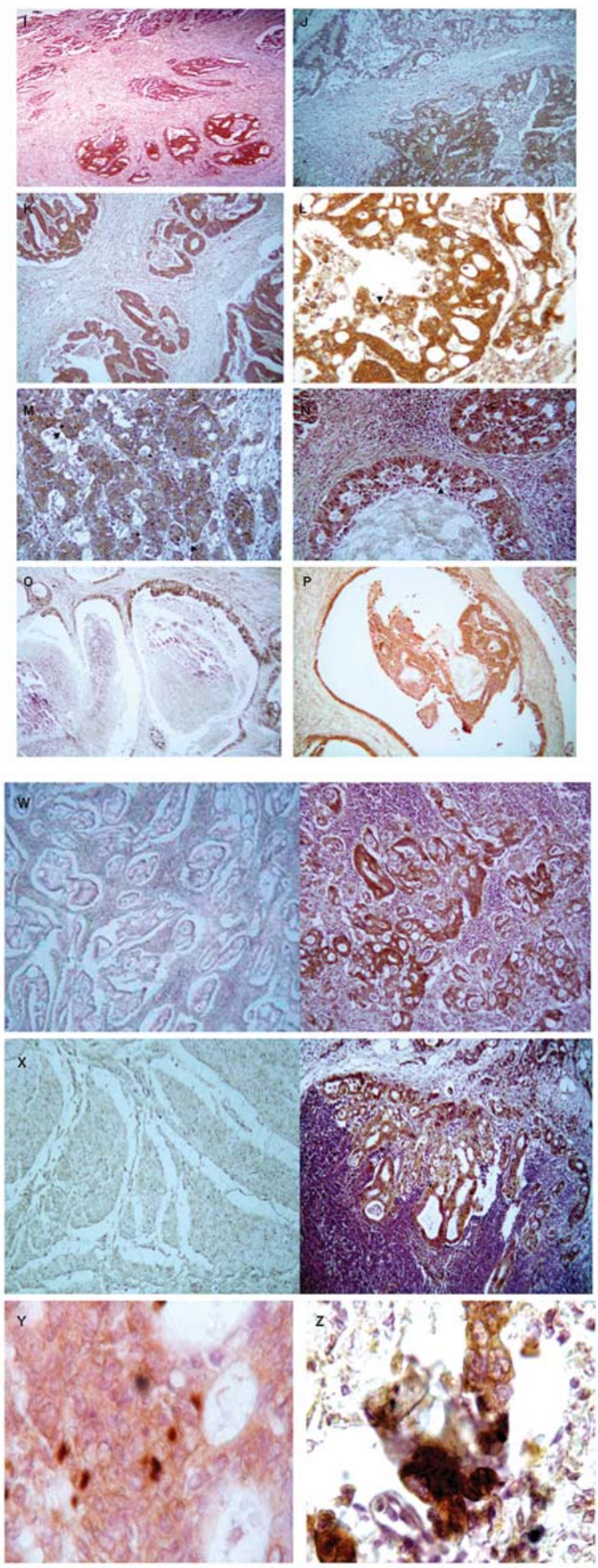

Figure 1. Representative images of immunohistochemical staining for OPRT in CRCs. (A-C) Immunohistochemical staining for OPRT in non-neoplastic colorectal epithelium. (A) In normal epithelium, granular staining is observed only in the crypt (arrowhead). (B) In crypt adenoma, weak, patchy staining is visible. (C) At the boundary area between the carcinoma and normal epithelium, only carcinoma cells show dense cytoplasmic staining (upper side). (D-G) Representative images of IHC for OPRT in mucosal carcinoma lesions. (D) In one case, 50 \% of tumor cells show irregular cytoplasmic staining in mucosal carcinoma lesions of welldifferentiated adenocarcinoma (case 11). (E) Most tumor cells in mucosal carcinoma lesions show diffuse cytoplasmic stainining for OPRT (case 10). (F and G) Tumor cells in mucosal carcinoma lesions are negative for OPRT (F, case 51; G, case 54). (H-J) Infiltrative components show dense staining for OPRT, whereas corresponding mucosal carcinoma lesions do not express OPRT (H, case 33; I, case 43; J, case 34). (K-Q) Representative images of IHC for OPRT in infiltrative components. (K) Infiltrative components comprising well-differentiated adenocarcinoma show diffuse cytoplasmic staining (case 44). (L-N) High-grade images of diffuse cytoplasmic staining with nuclear staining (arrowheads) in moderately-differentiated adenocarcinoma (L, case 2), poorly-differentiated adenocarcinoma (M, case 16), and mucinous carcinoma (N, case 55). (O-Q) Infiltrative components comprising of mucinous carcinoma show diffuse staining for OPRT (O, case 49; $\mathrm{P}$, case 21; Q, case 4). (R-Z) Representative images of IHC for OPRT in lymphovascularly invasive lesions and metastatic lymph nodes. (R-T) Dense cytoplasmic staining with nuclear staining (arrowhead) is observed in lymphovascularly invasive lesions (R, case 2; S, case 15; T, case 38). (U) In the case of metastatic lymph nodes, neoplastic tubule-specific expression is observed (case 2). (V) In the case of metastatic lymph nodes comprising mucinous carcinoma, only thin, patchy staining is visible, which are considered negative (case 4). (W and X) Dense staining is observed in the metastatic lymph node (right column), whereas no staining is detected in corresponding primary lesions (left column) (W, case 20; X, case 15). (Y and Z) High-grade image of nuclear staining in the metastatic lymph nodes (case 21). 
Table IV. The expression rate of OPRT in lymphovascular invasion $(n=34)$ and metastastic lymph nodes $(n=17)$.

\begin{tabular}{lcc}
\hline Expression grade & Ly or V & Lymph nodes \\
\hline 0 & 3 & 0 \\
$1^{+}$ & 1 & 2 \\
$2^{+}$ & 20 & 10 \\
$3^{+}$ & 10 & 5 \\
Expression rate & & $15 / 17(88.2 \%)$ \\
$\left(2^{+}\right.$or $\left.3^{+}\right)$ & $30 / 34(88.2 \%)$ & $7 / 15(46.6 \%)$ \\
Mucosal (-) rate & $4 / 30(13.3 \%)$ & $3 / 15(20.0 \%)$ \\
Infiltrative (-) rate & $1 / 30(3.3 \%)$ & \\
\hline
\end{tabular}

Ly or V, lymphovascularly invasive lesions; lymph nodes, metastatic lymph nodes; expression grade 0 , undetectable staining or weak, patchy cytoplasmic staining; $1^{+}$, focal staining in $<25 \%$ of tumor cells; $2^{+}$, diffuse, granular staining in $>50 \%$ of tumor cells; $3^{+}$, diffuse cytoplasmic staining with dense nuclear staining; mucosal (-), the case in which corresponding mucosal carcinoma lesions in primary tumors do not express OPRT, although OPRT is expressed in lymphovascularly invasive lesions or metastatic lymph nodes and infiltrative (-), the case in which corresponding infiltrative lesions in primary tumors do not express OPRT, although OPRT is expressed in lymphovascularly invasive lesions or metastatic lymph nodes.

Table V. A comparison of the expression rate between each component.

\begin{tabular}{lcc}
\hline Component & $\begin{array}{c}\text { OPRT }(-) \\
\left(0,1^{+}\right)\end{array}$ & $\begin{array}{c}\text { OPRT(+) } \\
\left(2^{+}, 3^{+}\right)\end{array}$ \\
\hline Mucosal & 38 & $18(32.1 \%)$ \\
Infiltrative & 16 & $37(69.8 \%)$ \\
Ly or V & 4 & $30(88.2 \%)$ \\
$\mathrm{N}$ & 2 & $15(88.2 \%)$ \\
\hline
\end{tabular}

Mucosal, mucosal carcinoma lesions; infiltrative, infiltrative lesions; Ly or $\mathrm{V}$, lymphovascularly invasive lesions and $\mathrm{N}$, metastatic lymph nodes.

significantly increased in metastatic CRCs and that nuclear staining of CLDN1 was observed in $35 \%$ of the metastatic lymph nodes, whereas membranous staining was observed in corresponding primary CRCs. The change in the pattern of expression of CLDN1 from primary to metastatic CRCs resembles the change observed for OPRT. To confirm whether OPRT and CLDN1 mislocalize during the progression of CRCs from primary sites to metastatic lymph nodes, we analyzed CLDN1 by IHC with a polyclonal anti-CLDN1 antibody (ready to use, Genetex, Inc.). Four pairs of primary CRC and corresponding metastatic lymph nodes, in which OPRT was diffusely expressed in metastatic lymph nodes with no staining in corresponding mucosal carcinoma
Table VI. A comparison of the expression patterns between CLDN1 and OPRT in four cases.

\begin{tabular}{ccc}
\hline Case number & $\begin{array}{c}\text { Mucosal } \\
\text { CLDN1/OPRT }\end{array}$ & $\begin{array}{c}\mathrm{N} \\
\text { CLDN1/OPRT }\end{array}$ \\
\hline 9 & $2^{+} / 1^{+}$ & $3^{+} / 3^{+}$ \\
20 & $1^{+} / 0$ & $2^{+} / 2^{+}$ \\
21 & $2^{+} / 1^{+}$ & $2^{+} / 2^{+}$ \\
24 & $2^{+/ 0}$ & $2^{+} / 2^{+}$ \\
\hline
\end{tabular}

Mucosal carcinoma lesions; $\mathrm{N}$, corresponding metastatic lymph node 0 , undetectable staining or weak, patchy cytoplasmic staining; $1^{+}$, focal staining in $<25 \%$ of tumor cells; $2^{+}$, diffuse, granular staining in $>50 \%$ of tumor cells and $3^{+}$, diffuse cytoplasmic staining with dense nuclear staining.

lesions. Of the four cases, three showed diffuse staining for CLDN1, which was limited to the cell membrane in mucosal carcinoma components (Fig. 2A-E). Furthermore, corresponding metastatic lymph nodes diffusely expressed CLDN1 (Fig. 2F). Nuclear staining was observed in one case (Fig. 2G). Notably, OPRT and CLDN1 showed nuclear staining in the same case (Fig. 2H and I).

\section{Discussion}

One of the notable features of our IHC analyses is that the patterns of expression of OPRT in CRCs were assessed individually for several different cancerous components, including primary mucosal carcinoma components, infiltrative lesions, lymphovascularly invasive components and metastatic lymph nodes. Our results yielded four major findings. The expression of OPRT in mucosal or infiltrative carcinoma lesions of primary CRCs correlates significantly with the presence of lymphovascular invasion. Furthermore, OPRT expression increases significantly during the process of cancer progression from mucosal carcinoma lesions to infiltrative lesions through lymphovascularly invasive lesions and metastatic lymph nodes. Additionally, in some cases, infiltrative components express OPRT, although the corresponding mucosal carcinoma do not express OPRT. Tumor cells of lymphovascularly invasive components and metastatic lymph nodes show nuclear staining of OPRT.

Several recent studies of OPRT in CRCs have focused mainly on the role of OPRT in response to 5-FU-based chemotherapy (4,5,7-9). Activity assay and RT-PCR studies have revealed that survival is significantly better for patients expressing high levels of OPRT (activity or mRNA levels) among CRC patients who underwent 5-FU-based adjuvant chemotherapy. There are few reports on IHC analyses of OPRT in CRCs. Tokunaga et al (4) studied 150 cases of CRCs and found that OPRT expression associates negatively with CRC progression and relates with a better prognosis and that patient survival rates are higher in OPRT-positive patients than in OPRT-negative patients by IHC. Tokunaga et al also reported that OPRT expression correlates negatively with progression in venous invasion. These results are not 

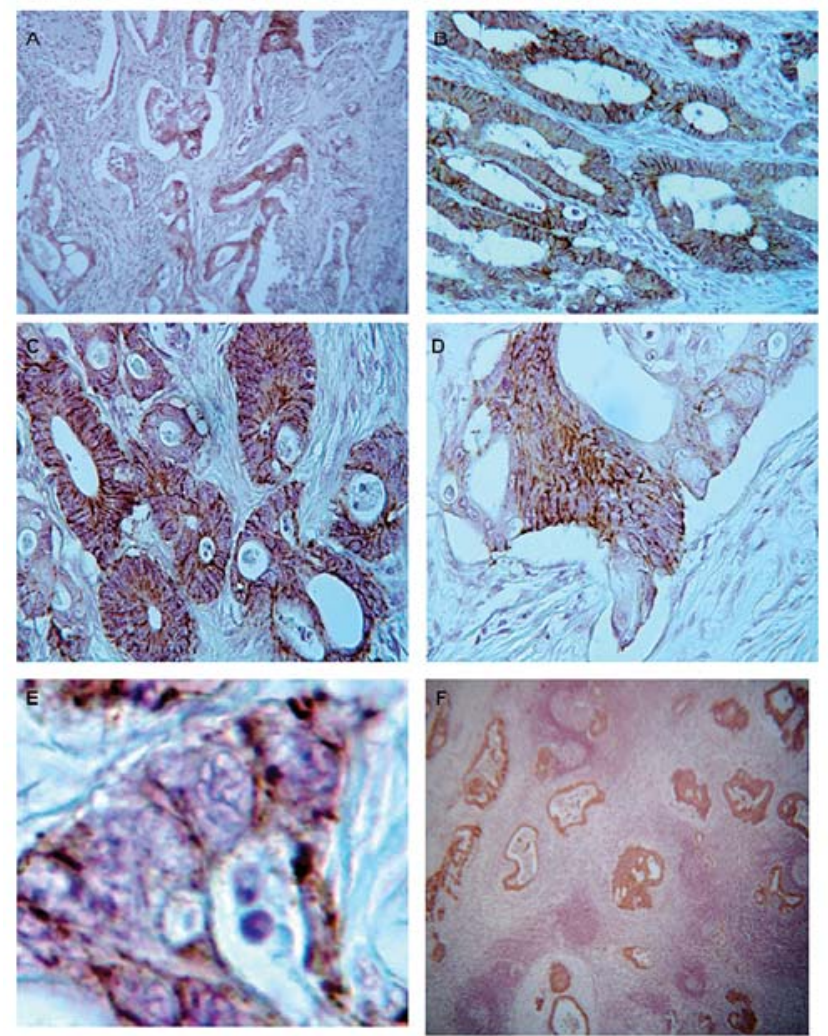
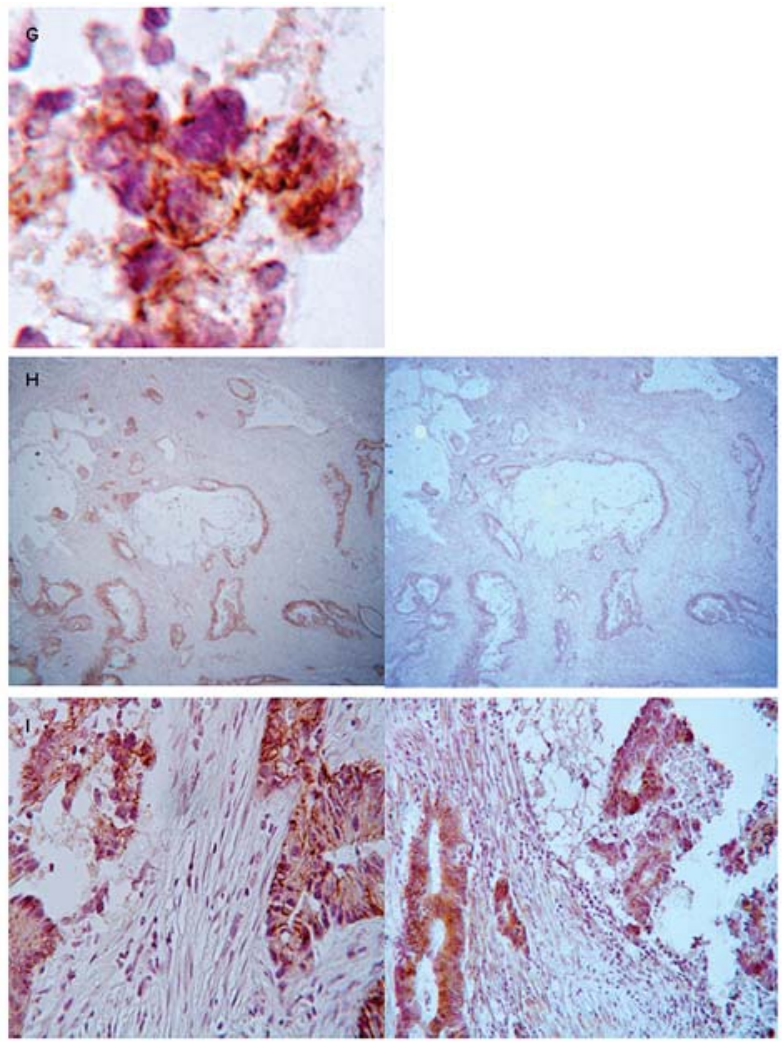

Figure 2. Representative images of IHC for CLDN1 in CRCs. (A) Thin membranous staining is visible 20\% of the primary tumors (case 9). (B-D) Dense membranous staining is observed in most tumor cells of primary lesions (B, case 20; C, case 21; D, case 24). (E) A high-grade image of membranous expression of CLDN1 in primary lesions (case 21). (F) Diffuse staining for CLDN1 in metastatic lymph nodes (case 9). (G) High-grade image of nuclear staining of CLDN1 in metastatic lymph nodes (case 9). (H and I) In one case (case 9), carcinoma cells in the primary lesions show membranous staining for CLDN1 (H, left column), where OPRT is focally expressed (1+) (H, right column). In corresponding metastatic lymph nodes, both CLDN1 (I, left column) and OPRT (I, right column) are diffusely expressed in the nuclei.

consistent with our present data. This discrepancy may originate from $\sim 3$-fold larger number of cases in the study by Tokunaga et al. However, another important difference is the method used to examine the expression status. Tokunaga et al reported that a case was identified as OPRT-positive or -negative without detailed morphological descriptions. Although the number of cases was smaller in our study, IHC allowed the visualization of diffuse expression of OPRT in infiltrative components of CRCs. In particular, in metastatic lymph nodes and lymphovascularly invasive components, most tumor cells showed dense staining of OPRT, despite the fact that some of these cases did not express OPRT in corresponding mucosal carcinoma components.

In previous reports, the presence of lymphovascular invasion and lymph node metastasis has been compared statistically with the pattern of OPRT expression in the primary tumor $(4,9)$. Our results clearly show that OPRT is involved in invasion and/or lymph node metastasis of CRC. In the present study, infiltrative lesions in primary tumors $(\mathrm{n}=53)$ was 2 -fold higher the frequency of OPRT expression $(69.8 \%)$ than that in mucosal carcinoma lesions $(32.1 \%)$. In 7 of the 53 cases, infiltrative lesions were composed mainly of mucinous carcinoma, six $(85.7 \%)$ of which showed diffuse staining of OPRT. In general, in CRCs, mucinous carcinoma shows lower chemosensitivity than other subtypes. Fujii et al (7) reported that the OPRT activity of mucinous carcinoma is significantly lower than that of other histological subtypes.
There are two possible explanations for this discrepancy. In the present study, corresponding mucosal carcinoma components comprised well- or moderately-differentiated adenocarcinoma in all seven cases, suggesting that so-called mucinous carcinoma, mucinous components involving throughout the tumor, have biological features different from those of mucinous carcinoma components analyzed in the present study. Furthermore, mucinous carcinoma is characterized by nodular extracellular mucin lakes with scanty malignant epithelial cells. The low OPRT activity value in a study by Fujii et al may be attributed to the low density of cells in the mucinous carcinoma tissues examined. We assume that the diffuse staining of OPRT in mucinous carcinoma lesions in infiltrative components does not represent a subtype specificity of OPRT. It is reasonable to speculate that the diffuse staining observed in the six cases represents only an association between OPRT expression and infiltration of CRC.

Since OPRT is involved in the first step of 5-FU metabolism, most previous studies reported that OPRT is a predictor of chemosensitivity or a better prognosis after adjuvant chemotherapy for several gastrointestinal carcinomas including CRC. Ichikawa et al (9) reported that the expression of OPRT mRNA might be a useful predictive parameter for the efficacy of fluoropyrimidine-based chemotherapy for metastatic CRCs. Ochiai et al (5) reported that patients with high OPRT activity had a better prognosis after 5-FU-based adjuvant chemotherapy. However, previous studies yielded 
several conflicting points. It is not reasonable to examine the association between the expression or enzyme activity of OPRT and chemosensitivity with samples from a case series with postoperative adjuvant chemotherapy. In the case of adjuvant chemotherapy, it is unclear whether prognosis is dependent on chemotherapy or the biological behavior of the tumor. Additionally, for analyses of chemosensitivity of recurrent CRCs, an analysis of the expression in primary lesions is not sufficient for the estimation of chemosensitivity of the recurrent tumor. Instead, we should use samples of the metastatic lesions, to which anti-cancer drugs behave directly. In the present case series, a diffuse expression of OPRT was observed in metastatic or invasive lesions of CRCs. Fujii et al (7) also reported that OPRT activity is increased in tumor cells with a higher Ki-67 labeling index. Fujii et al assumed that OPRT activity is involved in the rapid proliferation of tumor cells. In addition, some researchers showed that OPRT activity is high in metastatic lesions of CRCs, including lymph nodes and liver metastases $(10,11)$.

It is generally accepted that anti-cancer agents are more effective against rapidly proliferating, highly malignant tumors. Given the importance of OPRT in the activation of 5-FU, which influences chemosensitivity of a tumor, the present immunolocalization data for OPRT enhances our understanding of the significance and function of OPRT in the development, progression and treatment of cancer. In order to evaluate the significance of OPRT as a marker of chemosensitivity, analyses of OPRT expression metastatic components and a comparison between pre- and postchemotherapeutic tissue samples are needed.

In the present study, we found that OPRT expression is increased in lymphovascular invasive lesions and metastatic lymph nodes and also that OPRT is mislocalized from the cytoplasm to the nucleus in certain tumor cells in these components. We reported that OPRT shows similar mislocalization in hepatocellular carcinoma.

In 2005, Dhawan et al (6) reported the frequent nuclear translocation of CLDN1 in metastatic CRCs. Claudins are recently identified proteins, that are integral to the structure and function of tight junctions. In normal colorectal tissues and primary CRCs, CLDN1 expression is localized in the cell membrane. Dhawan et al assumed that this change in CLDN1 localization from primary to metastatic lesions of $\mathrm{CRCs}$ is induced by $\beta$-catenin/Tcf signaling. In addition, Shiou et al (12) reported that CLDN1 and Smad-4 expression correlated inversely in human CRC samples and that the activity of the CLDN1 promoter is repressed by Smad-4 transfection in human CRC cell lines. The most important point of these studies is that the nuclear translocation of a transmembrane protein is due to an upstream mechanism involved in oncogenic signaling, such as the $\beta$-catenin/Tcf pathway. In the present study, four pairs of primary CRC and corresponding metastatic lymph nodes were examined by CLDN1 IHC. In the first case, the nuclear localization of CLDN1 and OPRT was observed in the metastatic lymph node. It is not appropriate to assert that nuclear localization of OPRT is explained by a mechanism similar to that of CLDN1. However, our data suggest that the translocation of OPRT from the cytoplasm to the nucleus is induced by an upstream molecular oncogenic pathway. To confirm this, further functional analyses with CRC cell lines are needed.

\section{References}

1. Sakamoto K, Sugimoto Y, Miyadera K, et al: Preparation of anti-orotate phosphoribosyltransferase antibody and its application to immunohistochemical detection in human tumor cells. Int J Mol Med 16: 245-249, 2005

2. Ishida H, Shirakawa K, Ohsawa T, et al: Expression of mRNA levels of thymidylate synthase, dihydropyrimidine dehydrogenase, and orotate phosphoribosyltransferase of colorectal cancerrelationship among mRNA expression with response to 5-FU based treatment. Gan To Kagaku Ryoho 32: 1929-1934, 2005 (in Japanese).

3. Sanada Y, Yoshida K, Ohara M, et al: Expression of orotate phosphoribosyltransferase (OPRT) in hepatobiliaryand pancreatic carcinoma. Pathol Oncol Res 13: 105-113, 2007.

4. Tokunaga Y, Sasaki H and Saito T: Clinical role of orotate phosphoribosyltransferase and dihydropyrimidine dehydrogenase in colorectal cancer treated with postoperative fluoropyrimidine. Surgery 141: 346-353, 2007

5. Ochiai T, Nishimura K, Noguchi H, et al: Prognostic impact of orotate phosphoribosyltransferase activity in respectable colorectal cancer treated by 5 -fluorouracil-based adjuvant chemotherapy. J Surg Oncol 94: 45-50, 2006.

6. Dhawan P, Singh AB, Deane NG, et al: Claudin-1 regulates cellular transformation and metastatic behavior in colon cancer. J Clin Invest 115: 1765-1776, 2005.

7. Fujii R, Seshimo A and Kameoka S: Relationships between the expression of thymidylate synthase, dihydropyrimidine dehydrogenase, and orotate phosphoribosyltransferase and cell proliferative activity and 5-fluorouracil sensitivity in colorectal carcinoma. Int J Clin Oncol 8: 72-78, 2003.

8. Kuramochi H, Hayashi K, Uchida K, et al: 5-Fluorouracil-related gene expression levels in primary colorectal cancer and corresponding liver metastasis. Int J Cancer 119: 522-526, 2006.

9. Ichikawa W, Uetake H, Shirota $Y$, et al: Both gene expression for orotate phosphoribosyltransferase and its ratio to dehydropyrimidine dehydrogenase influence outcome following fluoropyrimidine-based chemotherapy for metastatic colorectal cancer. Br J Cancer 89: 1486-1492, 2003.

10. Matsusaka S, Yamasaki H, Fukushima M, et al: Upregulation of enzymes metabolizing 5-fluorouracil in colorectal cancer. Chemotherapy 53: 36-41, 2006.

11. Inokuchi M, Uetake H, Shirota Y, et al: Gene expression of 5fluorouracil metabolic enzymes in primary colorectal cancer and corresponding liver metastasis. Cancer Chemother Pharmacol 53: 391-396, 2004.

12. Shiou SR, Singh AB, Moorthy K, et al: Smad4 regulates claudin- 1 expression in a transforming growth factor- $\beta$ independent manner in colon cancer cells. Cancer Res 67: 1571-1579, 2007. 